\title{
Post-Pneumonectomy ARDS and Ogilvie Syndrome - A Case Report
}

\author{
Radu T. Stoica1,2* Ioan Cordoș ${ }^{1,3}$, Anca Macri1 $^{1}$ \\ 1 "Marius Nasta" Pneumophtisiology Institute, Bucharest, Romania \\ 2 Faculty of Medicine, "Titu Maiorescu" University, Bucharest, Romania \\ 3 "Carol Davila" University of Medicine and Pharmacy, Bucharest, Romania
}

\begin{abstract}
Introduction: The Acute Respiratory Distress Syndrome (ARDS) is a severe hypoxemic acute lung injury that may complicate lung resections. Reported mortality is very high, up to $50 \%$. This report covers an ARDS case occurring post left pneumonectomy, with a favorable outcome, despite association with an acute colonic pseudo-obstruction syndrome (Ogilvie syndrome) that required abdominal surgery for decompression.
\end{abstract}

Case report: A 60-year old Caucasian male, diagnosed with a stage IIIA left lung tumor underwent a left pneumonectomy. On the second postoperative day, the patient developed ARDS, requiring ventilatory support. Two days later, as the multiple organ dysfunction worsened, a bowel obstruction occurred. With an acute colonic pseudo-obstruction diagnosis, the decision was to perform laparotomy and a temporary cecostomy. A subsequent improvement in the respiratory parameters and vital functions resulted in weaning from ventilator 8 days after the onset of the ARDS and transfer to the surgery ward 14 days after pneumonectomy.

Keywords: post-pneumonectomy ARDS, Ogilvie syndrome, acute colonic pseudo-obstruction, cecostomy

Received: 4 November 2017 / Accepted: 30 January 2018

\section{- INTRODUCTION}

The Society for Thoracic Surgeons General Thoracic Surgery Database of the USA reports a 3.1\% [1] postpneumonectomy ARDS incidence with a reported mortality exceeding $50 \%$ in most statistics $[1,2]$. Any associated major complication (renal, cardiovascular, digestive etc.) contributes to a modest prognosis for these patients [3].

\section{CASE REPORT}

A 60-year old Caucasian male, in good general condition, with a history of smoking of 42 packs a year, was diagnosed with a lung tumor in his left upper lobe, accompanied by hemoptysis. The fibrobronchoscopy and $\mathrm{X}$-ray revealed a non-small cell bronchogenic carcinoma, apparently localized in the left upper lobe (T2b at the lung CT-scan). The BMI was 27 and routine hematological and biochemical parameters were within nor- mal limits. $\mathrm{FEV}_{1}$ was 2.4 / 3.01 liters (76\% from predicted). The carbon monoxide transfer factor - TLCO was $74 \%$ from predicted, the six-minute walk test (6MWT) and the arterial blood gases $\left(\mathrm{PaO}_{2} 78 \mathrm{mmHg}, \mathrm{PaCO}_{2}\right.$ $37 \mathrm{mmHg}$ ) were all within normal limits. Echocardiography revealed a left EF of $48 \%$, with no pulmonary hypertension or valvular heart disease. Given the physical status score of ASA III and the Thoracic Revised Cardiac Risk Index of 1.5, the indication was for general anesthesia on a single lung, combined with thoracic epidural analgesia.

The left thoracotomy revealed that the tumor, located in the upper left lobe, spread to the apical segment of the left lower lobe and penetrated to the distal segment of the left pulmonary artery. Therefore, the decision was made to perform a left extrapericardial pneumonectomy, with lymphadenectomy, which went entirely uneventful.

Anesthesia. After an epidural catheter was placed at T4-T5 level, the general anesthesia was induced with 
$0.3 \mu \mathrm{g} / \mathrm{kg}$ fentanyl, $2.2 \mathrm{mg} / \mathrm{kg}$ propofol and $0.6 \mathrm{mg} / \mathrm{kg}$ rocuronium. The patient was intubated with a leftsided double-lumen tube 39 French, inserted using a fibrobronchoscope. Low-flow anesthesia was maintained with MAC 1-1.2 sevoflurane with $\mathrm{FIO}_{2} 0.6$ of oxygen, combined with intra-venous Fentanyl and rocuronium. After an initial recruitment manoeuvre, made through an inflation pressure of $40 \mathrm{~cm} \mathrm{H}_{2} \mathrm{O}$ sustained for 15 seconds, the ventilation was on pressurecontrolled mode with a plateau pressure (Pplat) of 16 $\mathrm{cm} \mathrm{H}_{2} \mathrm{O}$ and a positive end-expiratory pressure (PEEP) of $6 \mathrm{~cm} \mathrm{H} \mathrm{H}_{2} \mathrm{O}$. The tidal volume of $6 \mathrm{ml} / \mathrm{Kg}$, was maintained with a driving pressure of $10 \mathrm{~cm} \mathrm{H}_{2} \mathrm{O}$ during one-lung ventilation (OLA), without repetitive pulmonary inflation.

The patient was extubated in the operating room, conscious, with normal respiratory and hemodynamic parameters and transferred to the postoperative ward.

Epidural analgesia consisted of a mixture of $0.3 \%$ ropivacaine and 0.1 micrograms fentanyl/ $50 \mathrm{ml}$. During the 3-hour surgery, the patient received fluids at a rate of $8 \mathrm{ml} / \mathrm{kg} / \mathrm{h}(1.6 \mathrm{l})$ and $1 \mathrm{ml} / \mathrm{kg} / \mathrm{h}$ in the first 24 hours after surgery. The total fluid infusion given during surgery and in the first 24 postoperative hours was 3.31 (2.8 liters of crystalloid and 0.51 colloid fluid) with a net negative fluid balance of $0.5 \mathrm{l}$. About 36 hours later, dyspnea with hypoxemia sets in and after 4 hours of inefficient non-invasive ventilation, the trachea was intubated to facilitate mechanical ventilation.

In this case, the post-pneumonectomy, moderate ARDS diagnosis was supported by the clinical and radiological criteria [4].

Severe hypoxemia with onset in the first two postoperative days was accompanied by the presence of new infiltrates on the X-ray chest confirmed on the thoracic CT-scan (Figures 1, 2). Normal blood pressure, the lack of peripheral edema and normal biventricular function on transthoracic echocardiogram were elements in supporting the diagnosis of non-cardiogenic pulmonary edema.

The patient was ventilated with a standardized ARDSNet protocol that focused on low tidal volume $(5 \mathrm{ml} / \mathrm{kg}$ of ideal body weight and a target $\mathrm{pH}>7.25)$, with a positive end-expiratory pressure (PEEP) increased to $8 \mathrm{~cm} \mathrm{H}_{2} \mathrm{O}$, while holding a prone position for 18 hours. On the $4^{\text {th }}$ postoperative day, the patient develops an obstructive syndrome, with abdominal distension, gastric stasis (3 liters/12 hours) and complete shutdown of the bowel transit. Abdominal X-ray

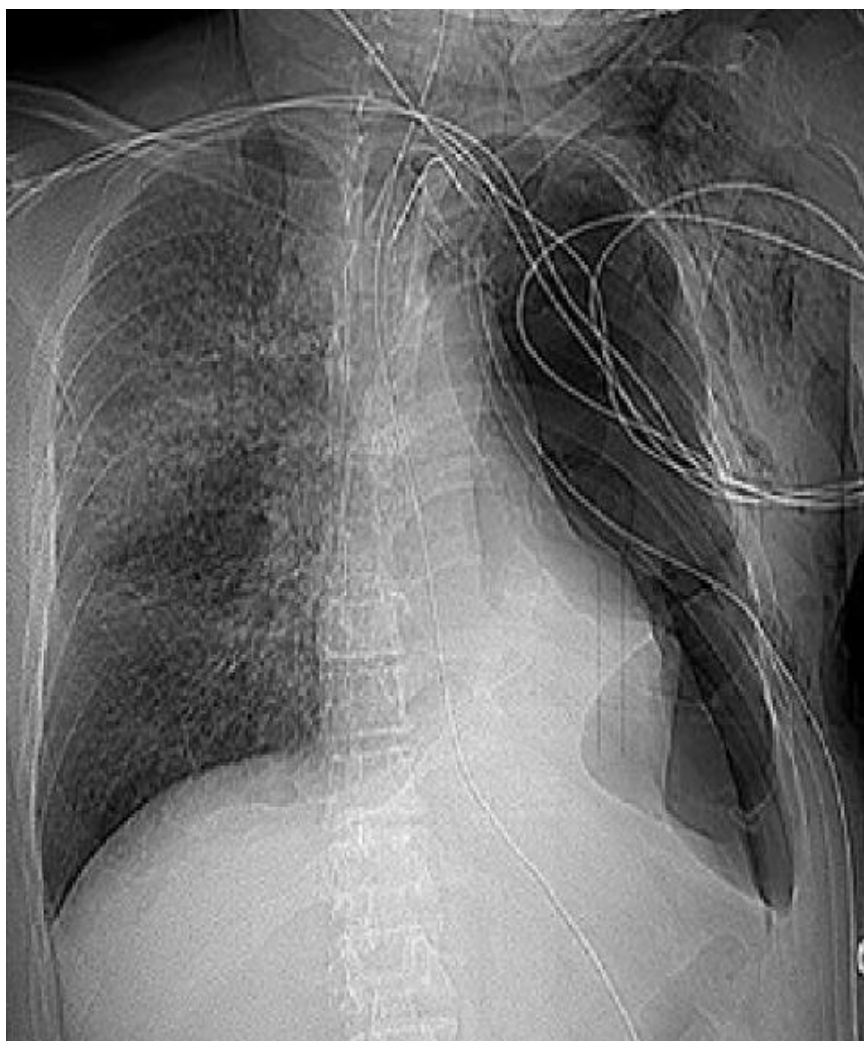

Fig. 1. Chest Rx. Diffuse coalescent opacities of the right lung.

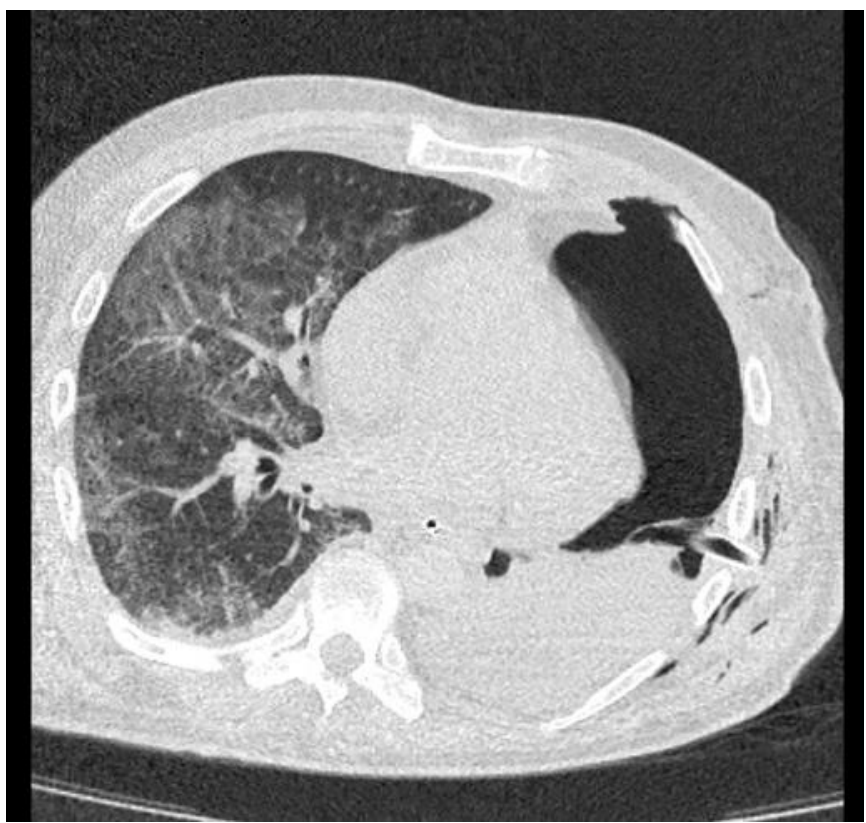

Fig. 2. Lung CT.Ground-glass opacification . Right lung ARDS.

reveals distended loops of bowel between the cecum and the sigmoid, with visible haustra. (Figure 3). The hypoxemia becomes refractory, the pulmonary compliance drops and, hemodynamically, the patient become hypotensive and tachycardic (120-130/min). 


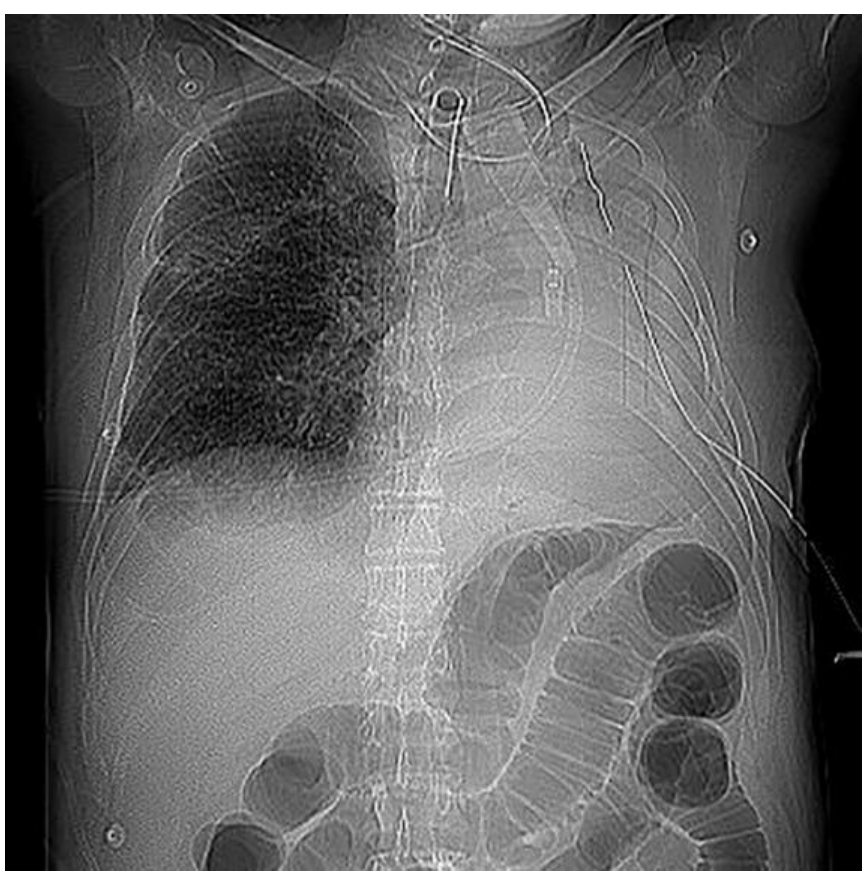

Fig. 3. Abdominal Rx. Distended loops of bowel with visible haustra.

The obstructive syndrome persists despite the conservative treatment. This consisted of maintaining the hydro-electrolyte balance, discontinuing the opioids, gastric aspiration, $2 \mathrm{mg}$ IV neostigmine repeated at 4 and 10 hours and placement of a decompressive rectal Faucher tube. Surgery was performed 20 hours from the onset of the abdominal obstructive syndrome, revealing a severe colic distension, without mechanical obstruction, with an approximately $12-\mathrm{cm}$ diameter cecum and impending diastatic perforation. Acute dilation of the colon and rarely of the small bowel in the absence of a mechanical bowel obstructive injury characterizes Ogilvie's syndrome or the acute colonic pseudo-obstruction (ACPO) [5]. Colic decompression was performed by a cecostomy with the insertion of a Petzer tube. The patient underwent a tracheotomy in order to facilitate aspiration, decrease the endotracheal tube resistance and the work of breathing [6]. Further evolution was favorable, the pulmonary compliance, ventilation pressure and gas exchanges being rapidly restored. Two days later, the ventilator weaning procedures were initiated. The cecostomy and tracheotomy were reversed 14 days after surgery.

\section{Discussions}

The risk factors most commonly associated with ARDS after pulmonary resections are age $>60$, male gender, chronic lung diseases, alcoholism, preoperative addon therapy, pneumonectomies, massive transfusions and excessive fluids during surgery [7]. In this respect, the case presented a medium risk (left pneumonectomy, gender, potentially chronic lung damage). The administration of fluids during surgery and on the first postoperative days was slightly restrictive, in accordance with the current recommendations [8]. The intraanesthetic strategy, including the single lung protective ventilation and the combined IV and epidural analgesia were additional measures intended to prevent acute lung injury. Protective mechanical ventilation, while holding a prone position for 18 hours, rarely used and reported in post-pneumonectomy ARDS cases [9], was efficient until the onset of the bowel obstruction.

The exact pathogenesis of the Ogilvie syndrome, first time described in 1948 [10] or ACPO is still debated. The imbalance of the enteric autonomic nervous system, with exaggerated parasympathetic over-activity, is the main culprit in the theory initially suggested by Ogilvie. Currently, also based on the pharmacological response in some cases, it is deemed that the high sympathetic tone and the low parasympathetic tone are responsible for the proximal colic obstruction and relaxation, respectively [11].

The syndrome occurs mainly in older patients, with multiple impairments, postoperative, in patients that are critically ill or under medication that affects bowel motility. In such patients, the clinical diagnosis is made by excluding other causes of bowel obstruction and by an abdominal radiological examination. A CT scan has a $90 \%$ sensitivity and specificity as it can detail an extensive colonic dilatation without an obstructive lesion at the intermediate transitional zone or adjacent to the splenic flexure [12].

The reported case includes a series of general risk factors for developing ACPO including major surgery, sedatives and opioid medication. Chest surgery has not been associated with this syndrome that occurs more frequently after abdominal-pelvic surgery [13]. The concomitant presence of post-pneumonectomy ARDS and ACPO has not described previously in the literature. The diagnosis was suspected exclusively based on the abdominal radiograph, as his severe hypoxemia did not allow for him to be moved for the CT scan.

The most severe complication that can occur is the diastatic perforation of the cecum, whose risk increases with the size of the cecal dilatation and the duration of the obstruction. Cecal dilatation over $12 \mathrm{~cm}$ and a colic 
distension exceeding 6 days are seen as the limits of the maximum risk of perforation [14].

Treatment is aimed at colic decompression, being either conservative or surgical. The most commonly used pharmacological treatment is neostigmine (reversible acetylcholinesterase inhibitor) or the antagonists of opioid receptors (methylnaltrexone), more rarely used [14]. The prone position with lifted hips helps eliminate the gases. Colic relief is done by nasogastric tube and rectal gas tube or by colonoscopy which, when possible, seems to have a significant benefit with relatively low risks but high relapse rate (over $40 \%$ ). The response to these maneuvers may be visible within 3 to 5 days. A delay in the therapeutic response and the cecal dilatation pose a risk of perforation and peritonitis. This is why the surgical solution is usually applied in cases that are serious or fail to respond to the conservative therapy. Cecostomy is more commonly performed, as it can be done either by percutaneous colonoscopy [15] or by laparotomy. In extreme cases, with ischemia or cecal perforation with peritonitis, right colectomy may be an option. Obviously, mortality is high in these cases, with $30 \%$ on a retrospective series of 179 ACPO cases [13].

In our case, the failure of the conservative therapy had to be quickly evaluated. The physiopathological consequences of the bowel obstruction on the breathing and the mechanical ventilation, as well as on the other vital functions, especially the cardiovascular and renal functions, required emergency colic decompression through cecostomy. The intervention saved the patient as the cecal dilatation was very large $(12 \mathrm{~cm})$ with a risk of imminent perforation, resulting in a favorable outcome, with rapid improvement in the vital functions and the general condition.

\section{CONCLUSIONS}

This is the first case report in the literature where two extremely severe conditions, relatively rare, are associated, namely post-pneumonectomy ADRS and the Ogilvie syndrome, both with high mortality rates. The early diagnosis of the two syndromes, as they occurred, the correct ICU measures and the immediate surgery (cecostomy) led to a favorable outcome for the patient.

\section{CONFLICT OF INTEREST}

None to declare.

\section{REFERENCES}

1. Shapiro M, Swanson SJ, Wright CD, et al. Predictors of Major Morbidity and Mortality After Pneumonectomy Utilizing The Society for Thoracic Surgeons General Thoracic Surgery Database. Ann Thorac Surg. 2010;90:927-35.

2. Ruffini E, Parola A, Papalia E, et al. Frequency and mortality of ARDS and acute respiratory distress syndrome after pulmonary resection for bronchogenic carcinoma. Eur J Cardiothorac Surg. 2001;20:30-7.

3. Ando K, Doi T, Moody SY, Ohkuni Y, Sato S, Kaneko N. The effect of comorbidity on the prognosis of acute lung injury and acute respiratory distress syndrome. Intern Med. 2012;51:1835-40.

4. Ferguson ND, Fan E Camporota $L$, et al. The Berlin definition of ARDS: an expanded rationale, justification, and supplementary material. Intensive Care Med. 2012;38:1573-82.

5. Durai R. Colonic pseudo-obstruction. Singapore Med J. 2009; 50:237-44.

6. Davis K Jr, Campbell RS, Johannigman JA, Valente JF, Branson RD. Changes in Respiratory Mechanics After Tracheostomy. Arch Surg. 1999;134:59-62.

7. Licker M, de Perrot M, Spiliopoulos A, et al. Risk factors for ARDS after thoracic surgery for lung cancer. Anesth Analg. 2003;97:1558-65.

8. Evans RG, Naidu B. Does a conservative fluid management strategy in the perioperative management of lung resection patients reduce the risk of acute lung injury? Interact Cardiovasc Thorac Surg. 2012;15:498-504.

9. Cornejo R, Romero C, Goñi D. Prolonged prone position ventilation for severe respiratory distress syndrome postpneumonectomy. Report of one case. Rev Med Chil. 2009;137:1351-56

10. Ogilvie H. Large-intestine colic due to sympathetic deprivation; a new clinical syndrome. Br Med J. 1948;2:671-3.

11. Wells Cl, O'Grady G, Bissett IP. Acute colonic pseudoobstruction: A systematic review of aetiology and mechanisms. World J Gastroenterol. 2017;23:5634-44.

12. Choi JS, Lim JS, Kim H, et al. Colonic pseudoobstruction: CT findings. AJR Am J Roentgenol. 2008;190:1521-26.

13. Vanek VW, Al-Salti M. Acute pseudo-obstruction of the colon (Ogilvie's syndrome). An analysis of 400 cases. Dis Colon Rectum. 1986;29:203-10.

14. Ben Ameur H, Boujelbene S, Beyrouti MI. Treatment of acute colonic pseudo-obstruction (Ogilvie's Syndrome). Systematic review. Tunis Med. 2013;91:565-72.

15. Arpana J, Vargas HD, Advances and Challenges in the Management of Acute Colonic Pseudo-Obstruction (Ogilvie Syndrome). Clin Colon Rectal Surg. 2012;25:37-45. 